\title{
Successful Treatment of Eccrine Porocarcinoma Metastasized to a Cervical Lymph Node with CyberKnife Radiosurgery
}

\author{
Taku Fujimura Akira Hashimoto Sadanori Furudate Yumi Kambayashi \\ Takahiro Haga Setsuya Aiba
}

Department of Dermatology, Tohoku University Graduate School of Medicine, Sendai, Japan

\section{Key Words}

Eccrine porocarcinoma $\cdot$ CyberKnife $\cdot$ Stereotactic radiotherapy

\begin{abstract}
Eccrine porocarcinoma is a rare type of skin cancer that originates from eccrine sweat glands or acrosyringium and mainly occurs in the elderly. In this report, we describe an 85-year-old Japanese woman with eccrine porocarcinoma that metastasized to a cervical lymph node who was cured with CyberKnife radiosurgery. Because our patient had a high risk of perioperative complication, standard surgical therapy with a wide margin was impractical. Our present study suggests the novel possibility of using CyberKnife for the treatment of inoperable metastatic porocarcinoma.

(c) 2014 S. Karger AG, Basel
\end{abstract}

\section{Introduction}

Eccrine porocarcinoma is a rare type of skin cancer originating from eccrine sweat glands or acrosyringium, representing $0.01 \%$ of all cutaneous tumors [1]. Since it mainly occurs in the elderly $[1,2]$, such patients may possess a high risk of perioperative complications and, in such cases, radical surgical treatment is not practical. In this report, we describe a case of recurrent eccrine porocarcinoma that metastasized to a cervical lymph node successfully treated with CyberKnife. 
Fujimura et al.: Successful Treatment of Eccrine Porocarcinoma Metastasized to a Cervical Lymph Node with CyberKnife Radiosurgery

\section{Case Report}

An 85-year-old woman consulted us with a 3-month history of a red nodule. On her initial visit, physical examination revealed a red, easy-to-bleed, dome-shaped nodule $20 \mathrm{~mm}$ in diameter on her right cheek (fig. 1a). Dermoscopy findings revealed irregular arborizing vessels, and whitish globular structures covered by prominent bleeding (fig. 1b). Skin biopsy revealed dense infiltration of clear, cuboidal, basaloid atypical cells throughout the dermis (fig. 2). Immunohistochemical staining revealed that these tumor cells were positive for CEA, 34bE12, p63, CK5/6 and CK14, and negative for GCDFP15. From these results, we diagnosed this case as eccrine porocarcinoma. We resected the tumor with a 2 -cm surgical margin. Six months later, lymph node swelling of the right cervical lesion was detected. We screened for a possible internal malignancy with positron emission tomography-computed tomography, which revealed significant enlargement of the cervical lymph nodes (fig. 3a). As the lesion was adjacent to the external carotid artery and standard surgical therapy would have been impractical, we employed a CyberKnife. The lesion of the cervical lymph node was irradiated with 50 Gy in 10 fractions. One month after the irradiation, the tumor mass had completely regressed, leaving a scar (fig. 3b). There was no severe side effect such as radiation dermatitis at the site of the irradiated lesion. There has been no sign of local recurrence or systemic lesions for 1 year.

\section{Discussion}

In this report, we describe a case of recurrent eccrine porocarcinoma that metastasized to a cervical lymph node successfully treated with CyberKnife. Porocarcinoma is described as a biologically aggressive neoplasm that frequently metastasizes to regional lymph nodes $[1,2]$. Indeed, Robson et al. [2] reported that approximately $20 \%$ of patients with porocarcinoma develop lymph node metastasis. The representative standard therapy for lymph node metastasis is regional lymphadenectomy, but its mortality rate is high [1-3]. In addition, since patients with porocarcinoma tend to be elderly [2], they may have a high risk of perioperative complications. Indeed, in our present case, since the lesion was located near critical facial structures and the patient was very old, radical surgical treatment such as cervical lymphadenectomy was not practical. In addition, conventional radiotherapy sometimes induces severe side effects after intensive treatment, and generally has been reported to be of little benefit in both primary and metastatic porocarcinoma, but has mostly been used as a palliative treatment [4]. For these reasons, we selected CyberKnife.

CyberKnife is a robotic image-guided stereotactic radiosurgery device that delivers a large ablative radiation dose to the tumor while sparing normal surrounding tissue. We previously reported successful treatment of a dermatofibrosarcoma protuberance and Merkel cell carcinoma using CyberKnife [5, 6]. As we previously reported [5, 6], this system is able to deliver extracranial stereotactic radiotherapy with millimetric precision, leading to disease control and toxicity profiles that are equal to or better than other available therapies [7]. To our knowledge, there is no English language report of metastatic porocarcinoma treated with CyberKnife without significant side effects. Our present study suggests the novel possibility of using CyberKnife for the treatment of inoperable metastatic porocarcinoma. Since porocarcinoma tends to recur both in local and remote areas long after the treatment and our follow-up period is short, further follow-up is needed for a definitive conclusion. 
Fujimura et al.: Successful Treatment of Eccrine Porocarcinoma Metastasized to a Cervical Lymph Node with CyberKnife Radiosurgery

\section{References}

1 Marone U, Caracò C, Anniciello AM, Di Monta G, Chiofalo MG, Di Cecilia ML, Mozzillo N: Metastatic eccrine porocarcinoma: report of a case and review of the literature. World J Surg Oncol 2011;9:32.

-2 Robson A, Greene J, Ansari N, Kim B, Seed PT, McKee PH, Calonje E: Eccrine porocarcinoma (malignant eccrine poroma): a clinicopathologic study of 69 cases. Am J Surg Pathol 2001;25:710-720.

3 Shiohara J, Koga H, Uhara H, Takata M, Saida T: Eccrine porocarcinoma: clinical and pathological studies of 12 cases. J Dermatol 2007;34:516-522.

4 Brown CW Jr, Dy LC: Eccrine porocarcinoma. Dermatol Ther 2008;21:433-438.

5 Hidaka T, Fujimura T, Hashimoto A, Aiba S: Successful treatment of pigmented dermatofibrosarcoma protuberance on glabella with CyberKnife radiosurgery. Acta Derm Venereol 2012;92:658-659.

6 Tuskada A, Fujimura T, Hashimoto A, Kambayashi Y, Furudate S, Haga T, Aiba S: Successful treatment of cutaneous Merkel cell carcinoma on eyelid with CyberKnife radiosurgery. Eur J Dermatol 2013;23:725-726.

7 Lartigau E, Mirabel X, Prevost B, Lacornerie T, Dubus F, Sarrazin T: Extracranial stereotactic radiotherapy: preliminary results with the CyberKnife. Onkologie 2009;32:209-215.
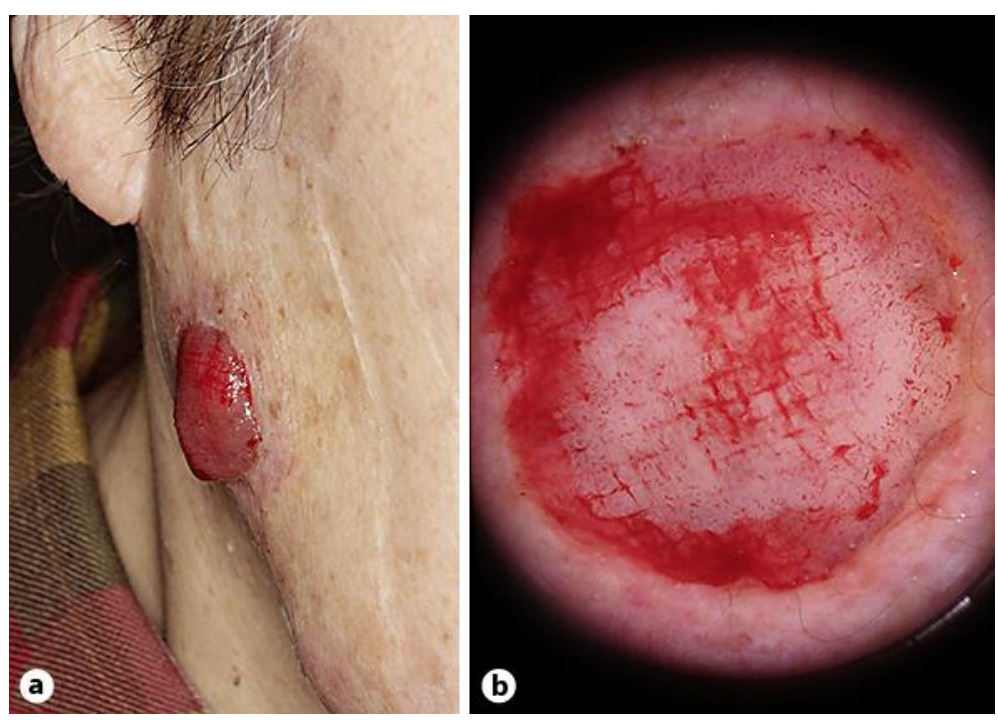

Fig. 1. a A red, easy-to-bleed, dome-shaped nodule $20 \mathrm{~mm}$ in diameter on the right cheek. b Dermoscopy findings revealed irregular arborizing vessels and whitish globular structures covered by prominent bleeding. 
Fujimura et al.: Successful Treatment of Eccrine Porocarcinoma Metastasized to a Cervical Lymph Node with CyberKnife Radiosurgery

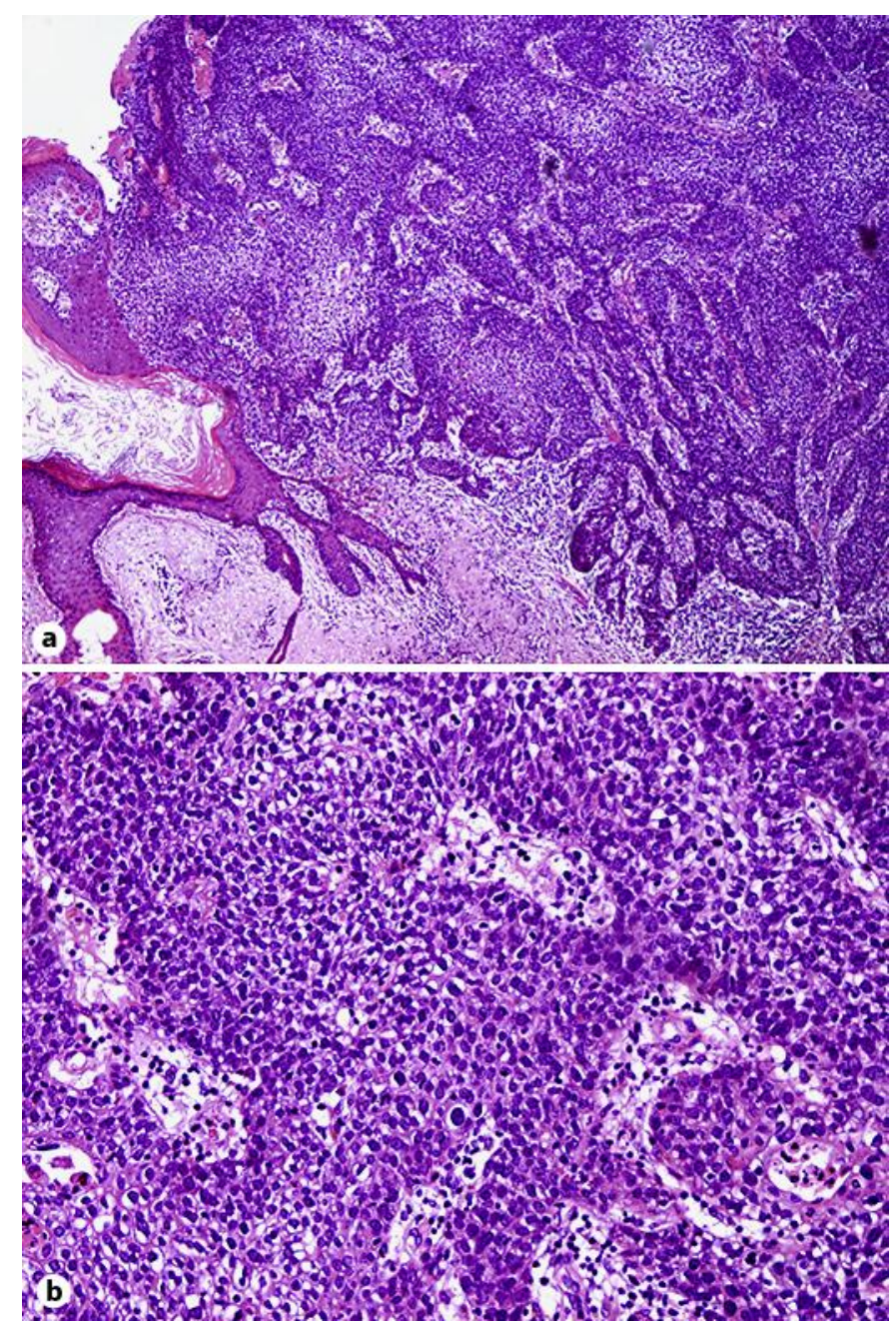

Fig. 2. Dense infiltration of clear, cuboidal, basaloid atypical cells infiltrating throughout the dermis. Original magnification: $\times 50(a), \times 200(b)$. 
Fujimura et al.: Successful Treatment of Eccrine Porocarcinoma Metastasized to a Cervical Lymph Node with CyberKnife Radiosurgery

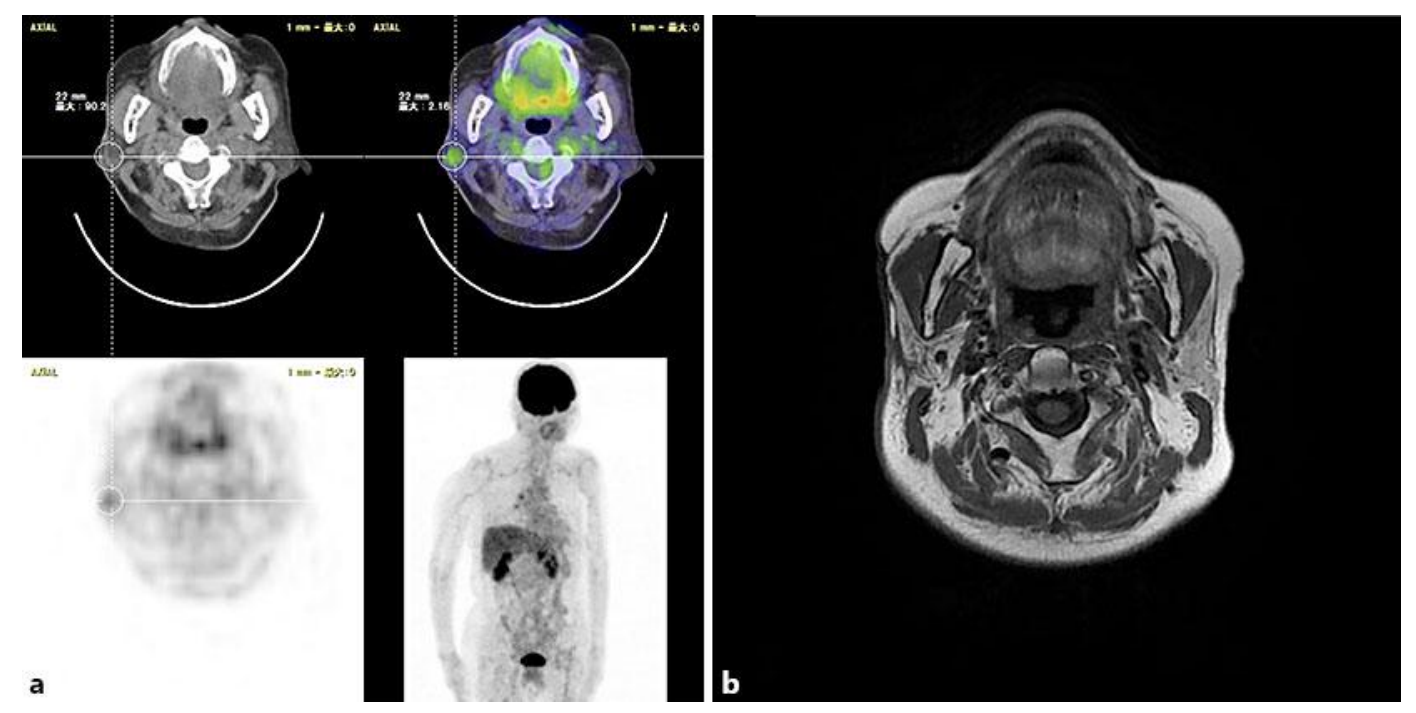

Fig. 3. a Positron emission tomography-computed tomography revealed significant enlargement of the cervical lymph nodes. b One month after the irradiation, follow-up computed tomography scan revealed that the tumor mass had completely regressed. 